ORIGINAL ARTICLE, PHARMACY

\title{
Spermicidal Constituents of Ethanolic Extract of Sacoglottis gabonensis Stem Bark
}

\author{
Gideon O. Alade, ${ }^{1,2}$, Jones O. Moody², Olanrewaju R. Awotona', Saburi A. Adesanya3, \\ Daowan Lai ${ }^{4}$, Peter Proksch ${ }^{5}$ \\ ${ }^{1}$ Department of Pharmacognosy \& Herbal Medicine, Faculty of Pharmacy, Niger Delta University, Wilberforce Island, Nigeria \\ 2 Department of Pharmacognosy, Faculty of Pharmacy, University of Ibadan, Ibadan, Nigeria \\ 3 Department of Pharmacognosy, Faculty of Pharmacy, Obafemi Awolowo University, lle-Ife, Nigeria \\ ${ }^{4}$ Department of Plant Pathology, College of Plant Protection, China Agricultural University, Beijing, PR China \\ ${ }^{5}$ Institute of Pharmaceutical Biology and Biotechnology, Heinrich Heine University, Dusseldorf, Germany
}

\section{Correspondence:}

Gideon O. Alade, Pharmacognosy

\& Herbal Medicine, Niger Delta University, Amassoma, Bayelsa Yenagoa, Bayelsa 560001, Nigeria E-mail: aladegideon@yahoo.com Tel: +2348067368038

Received: 05 July 2016

Accepted: 22 March 2017

Published Online: 09 May 2017

Published: 22 Dec 2017

Key words: sperm, immobilization, compound, Sacoglottis gabonensis

Citation: Alade GO, Moody JO, Awotona OR, Adesanya SA, Lai D, Proksch P. Spermicidal constituents of ethanolic extract of Sacoglottis gabonensis stem bark. Folia Medica 2017;59(4):437-42. doi: 10.1515/folmed-2017-0047
Aim: To isolate the spermicidal constituents of Sacoglottis gabonensis.

Materials and methods: The ethanolic extract with partitioned fractions of Sacoglottis gabonensis stem bark were subjected to sperm immobilization assay. The most active EtOAc fraction was further purified by column and Semi-Preparative High Performance Liquid Chromatography to give compounds which were characterized by spectroscopic methods (UV, LC/MS, and NMR). The compound(s) was also tested for sperm immobilization activity.

Results: The ethanolic extract showed $100 \%$ significant $(p<0.05)$ sperm immobilization activity at a concentration of $30 \mathrm{mg} / \mathrm{mL}$ at $20 \mathrm{~s}$ compared to both negative and positive controls. The most active ethyl acetate fraction yielded methyl 3,5-dihydroxy-4-methoxybenzoate, eriodictyol and bergenin. Bergenin had 100\% sperm immobilization activity at $20 \mathrm{mg} / \mathrm{mL}$ in $60 \mathrm{~s}$ which was significant $(p<0.05)$ also when compared to the positive and negative control while methyl 3,5-dihydroxy-4-methoxybenzoate, eriodictyol were not active.

Conclusion: The active spermicidal constituent in Sacoglottis gabonensis stem bark extract is bergenin. However, methyl 3,5-dihydroxy-4-methoxybenzoate and eriodictyol showed no activity. This plant is known for its aphrodisiac action; hence, caution may have to be exercised in its use because of its spermicidal effect.

\section{BACKGROUND}

Fertility control is an option in human population control. It is a global and national public concern. ${ }^{1}$ The majority of the currently available methods of fertility control are for women, participation of the male counterpart is poor. ${ }^{2}$ Some of the safe and effective male contraceptives currently known are condoms and vasectomy. ${ }^{3,4}$ Recently efforts are however, being made to explore the hidden wealth of medicinal plants for contraceptive use in males. ${ }^{5}$

In a preliminary work, Sacoglottis gabonensis stem bark has been previously reported for its sperm reducing potential in male rats with activity resident in the ethyl acetate fraction. ${ }^{6}$

\section{AIM}

This study aimed at isolating the compound(s) responsible for this activity.

\section{MATERIALS AND METHODS}

GENERAL EXPERIMENTAL PROCEDURES

${ }^{1} \mathrm{H},{ }^{13} \mathrm{C}$ and $2 \mathrm{D}$ NMR were recorded in deuterized solvents on Bruker DRX500 or AVANCE DMX 600 NMR spectrometers. Mass spectra were measured on a LC-MS Agilent 1100 series coupled with Thermoquest LCQ Deca XP (Finnigan). HPLC analysis was carried out on a Dionex UltiMate 3000 HPLC system (Thermo scientific) coupled to a photodiode array detector (DAD-3000RS). Routine detection 
was performed at $235,254,280$, and $340 \mathrm{~nm}$. The separation column $(125 \times 4 \mathrm{~mm}, \mathrm{~L} \times$ i.d. $)$ was prefilled with Eurospher-10 C18 (Knauer, Germany), and the following gradient was used $(\mathrm{MeOH} / 0.1 \%$ formic acid in water); $0 \mathrm{~min}, 10 \% \mathrm{MeOH} ; 5 \mathrm{~min}, 10 \%$ $\mathrm{MeOH} ; 35 \mathrm{~min}, 100 \% \mathrm{MeOH} ; 45 \mathrm{~min}, 100 \% \mathrm{MeOH}$ with a flow rate of $1.0 \mathrm{~mL} / \mathrm{min}$. HPLC separation was performed on a semi-preparative HPLC system of Lachrom-Merck Hitachi (Pump L7100 and UV detector L7400). The separation column $(300 \times 8 \mathrm{~mm})$ was prefilled with Europhere 100-C18 (Knauer, Germany) using a flow rate of $5.0 \mathrm{~mL} /$ min. Column chromatography was performed using Merck MN Silica gel 60 M (0.04-0.063 mm), Diaion HP20 (Mitsubishi Chemicals) or Sephadex LH20 (GE HealthCare) as stationary phases. For TLC analyses pre-coated Silica Gel 60 F254 plates (Merck) were used followed by detection under UV at 254 and $366 \mathrm{~nm}$ or after spraying with anisaldehyde reagent. All solvents were distilled prior to use, and spectral grade solvents were used for spectroscopic measurements.

\section{Plant material}

The stem bark of Sacoglottis gabonensis was collected and identified by Dr. A.T Oladele of the Department of Forestry and Wildlife Sciences, University of Port Harcourt, Rivers State of Nigeria. A voucher specimen (FHI109851) was deposited at the Herbarium of the Forestry Research Institute of Nigeria (FRIN), Ibadan, Nigeria.

\section{EXTRACTION AND ISOLATION}

The dried powder stem bark $(500 \mathrm{~g})$ of $S$. gabonensis was extracted with $80 \%$ cold ethanol and the extract was evaporated to give a brown residue $(25 \mathrm{~g})$. The suspension of the extract in nanopure water was subjected to solvent-solvent partitioning in a separating funnel with $n$-hexane $(400 \mathrm{~mL} \times 3)$, ethyl acetate $(400 \mathrm{~mL} \times 3)$, n-butanol $(500 \mathrm{~mL} \times 1)$, successively, and the various partitioned fractions were concentrated to dryness in vacuo using a rotary evaporator to obtain $1.3341 \mathrm{~g}$ of $\mathrm{n}$-hexane, $7.331 \mathrm{~g}$ ethyl acetate, $8.5091 \mathrm{~g}$ of $\mathrm{n}$-butanol and $5.0988 \mathrm{~g}$ of aqueous fractions, respectively. The fractions were monitored in HPLC coupled to an UV detector. $5.0 \mathrm{~g}$ of the ethyl acetate fraction was dissolved in dichloromethane and adsorbed onto silica gel. This was allowed to dry and chromatographed on silica gel under vacuum using a column $30 \mathrm{~cm}$ in height and $6 \mathrm{~cm}$ inner diameter with hexane containing increasing percentages (up to $10 \%$ ) of ethyl acetate, subsequently dichloromethane containing increasing percentages (up to 10\%) of methanol as eluent and each collected fraction was $500 \mathrm{~mL}$. Each fraction was collected, concentrated and monitored by HPLC. Eighty $\mathrm{mg}$ of fraction 2 was dissolved in a mixture of dichloromethane/methanol (50\%) and chromatographed over Sephadex LH-20 using an open column $(50 \mathrm{~cm} \times 4 \mathrm{~cm})$ and dichloromethane/methanol $(50 \%)$ as the eluent. The fraction was collected every 15 minutes using a fraction collector at a flow rate of 10 drops per second. One hundred and ten fractions were collected and pooled together with the aid of thin layer chromatography to get a total of seven fractions (1-7, respectively). Fractions 2, 4 and 6 were chosen for further purification. Due to low yields, fractions 1, 3, 5 and 7 couldn't be purified further. Each of the pooled fractions was dried using a rotary evaporator and also monitored by HPLC. Fraction 2 was finally purified using semi preparative HPLC to afford Compound 1. Fraction 4 was similarly processed using semi preparative HPLC to afford Compound 2. Fraction 6 was chromatographed on an open column $(50 \mathrm{~cm} \times 4 \mathrm{~cm})$ using HP-20 with methanol containing increasing percentages $(10 \%)$ of water as the eluent to afford a pure compound which came out as sub-fraction 3 (methanol : water; $2: 8$ ) and this afforded compound 3.

\section{SPERM IMMOBILIZATION ASSAY}

Healthy adult male albino rats were used for the experiments. The spermatozoa were obtained from the caudal epididymis excised from healthy adult rats after autopsy. The spermatozoa were collected into saline solution and adjusted to the concentration of $40-60 \times 10^{6}$ sperm $/ \mathrm{mL}$ and incubated in a water bath at $37^{\circ} \mathrm{C}$. In vitro spermicidal activity of various concentrations of the crude extract/fractions and compounds of Sacoglottis gabonensis was performed with each dilution, following the modified method of Sander and $\mathrm{Cramer}^{7}$ and the standard recommended by the $\mathrm{WHO}^{8}$. A stock solution of $120 \mathrm{mg} / \mathrm{mL}$ of the extract and the various fractions/ compounds was separately made and serial dilutions were made for the different concentrations to be employed. Sperm suspension $(0.5 \mathrm{~mL})$ was mixed with $0.5 \mathrm{~mL}$ of the different concentrations of the extract/fractions/compounds (7.5, 15, 30, 60 and 120 $\mathrm{mg} / \mathrm{mL}$ ) to evaluate the spermicidal activity. The two fluids were thoroughly mixed. Within 20,30 , 60,120 and 180 seconds of mixing, a drop of the mixture was immediately placed on warm slides and microscopically examined under $100 x$ to observe the 
motility of the spermatozoa. A mixture of $0.5 \mathrm{~mL}$ of sperm suspension and $0.5 \mathrm{~mL}$ of normal saline and cotton seed oil served as negative and positive controls, respectively. Each experiment was carried out in ten (10) replicates. Values were represented as mean \pm standard error of mean (SEM). One-way ANOVA with Tukey -Kramer Multiple Comparison Test was performed using GraphPad Prism version 5.01 for Windows, GraphPad Software, San Diego
California USA, www.graphpad.com. P values $<0.05$ were considered significant.

\section{RESULTS AND DISCUSSION}

\section{SPERMICIDAL ACTIVITY}

From the result (Table 1), S. gabonensis stem bark had $100 \%$ spermicidal activity at $120 \mathrm{mg} / \mathrm{mL}$ in $20 \mathrm{~s}$. This corroborates the $50 \%$ significant decrease in sperm count at $30 \mathrm{mg} / \mathrm{kg}$ in vivo in male rats. ${ }^{6}$

Table 1. In vitro sperm immobilization screening of Sacoglottis gabonensis, fractions and compound

\begin{tabular}{|c|c|c|c|c|c|c|c|}
\hline \multirow[b]{2}{*}{ S/No. } & \multirow[b]{2}{*}{$\begin{array}{l}\text { Plant extract / fractions / } \\
\text { compounds }\end{array}$} & \multirow[b]{2}{*}{$\begin{array}{c}\text { Concentration } \\
(\mathrm{mg} / \mathrm{mL})\end{array}$} & \multicolumn{5}{|c|}{ Spermicidal Activity / \% } \\
\hline & & & $20 \mathrm{~s}$. & $30 \mathrm{~s}$. & $60 \mathrm{~s}$ & $120 \mathrm{~s}$ & $180 \mathrm{~s}$ \\
\hline \multirow[t]{5}{*}{1} & Sacoglottis gabonensis extract & 7.5 & $74.50 \pm 2.63{ }^{*} \mathrm{a}, \mathrm{b}$ & $75.00 \pm 1.05^{*} \mathrm{a}, \mathrm{b}$ & $74.70 \pm 3.06^{* a, b}$ & $76.50 \pm 3.87^{* a, b}$ & $74.40 \pm 3.69^{* a, b}$ \\
\hline & & 15 & $75.60 \pm 4.79^{* a, b}$ & $74.40 \pm 2.68^{* a, b}$ & $76.50 \pm 6.89^{* a, b}$ & $76.40 \pm 4.55^{*} \mathrm{a}, \mathrm{b}$ & $74.00 \pm 2.84^{*} \mathrm{a}, \mathrm{b}$ \\
\hline & & 30 & $100.00 \pm 0.00^{* a, b}$ & $100.00 \pm 0.00^{* a, b}$ & $100.00 \pm 0.00^{* a, b}$ & $100.00 \pm 0.00^{* \mathrm{a}, \mathrm{b}}$ & $100.00 \pm 0.00^{*} \mathrm{a}, \mathrm{b}$ \\
\hline & & 60 & $100.00 \pm 0.00^{* a, b}$ & $100.00 \pm 0.00 * a, b$ & $100.00 \pm 0.00^{* a, b}$ & $100.00 \pm 0.00^{\mathrm{a}, \mathrm{b}}$ & $100.00 \pm 0.00^{*} \mathrm{a}, \mathrm{b}$ \\
\hline & & 120 & $100.00 \pm 0.00^{* a, b}$ & $100.00 \pm 0.00^{* a, b}$ & $100.00 \pm 0.00^{* a, b}$ & $100.00 \pm 0.00^{* a, b}$ & $100.00 \pm 0.00^{*} \mathrm{a}, \mathrm{b}$ \\
\hline \multirow[t]{5}{*}{2} & S. gabonensis hexane fraction & 7.5 & 0.00 & 0.00 & 0.00 & 0.00 & $20.00 \pm 2.36^{* a, b}$ \\
\hline & & 15 & 0.00 & 0.00 & 0.00 & 0.00 & $19.50 \pm 2.84^{*} \mathrm{a}, \mathrm{b}$ \\
\hline & & 30 & 0.00 & 0.00 & 0.00 & 0.00 & $19.50 \pm 4.38^{*} \mathrm{a}, \mathrm{b}$ \\
\hline & & 60 & 0.00 & 0.00 & 0.00 & 0.00 & $21.00 \pm 3.16^{* a, b}$ \\
\hline & & 120 & 0.00 & 0.00 & 0.00 & 0.00 & $19.50 \pm 1.58^{* a, b}$ \\
\hline \multirow[t]{5}{*}{3} & $\begin{array}{l}\text { S. gabonensis ethyl acetate } \\
\text { fraction }\end{array}$ & 7.5 & 0.00 & 0.00 & 0.00 & 0.00 & $40.00 \pm 3.33^{* a, b}$ \\
\hline & & 15 & 0.00 & 0.00 & 0.00 & 0.00 & $39.50 \pm 3.69^{*} \mathrm{a}, \mathrm{b}$ \\
\hline & & 30 & 0.00 & $20.00 \pm 2.33^{* a, b}$ & $60.00 \pm 2.36^{* a, b}$ & $59.50 \pm 2.84^{* a, b}$ & $100.00 \pm 0.00^{*} \mathrm{a}, \mathrm{b}$ \\
\hline & & 60 & $19.50 \pm 2.41^{* a, b}$ & $20.00 \pm 2.93^{* a, b}$ & $59.00 \pm 2.12^{*} \mathrm{a}, \mathrm{b}$ & $60.00 \pm 3.33^{* a, b}$ & $100.00 \pm 0.00^{*} \mathrm{a}, \mathrm{b}$ \\
\hline & & 120 & $20.50 \pm 2.40^{* a, b}$ & $38.50 \pm 2.42^{*_{\mathrm{a}, \mathrm{b}}}$ & $60.50 \pm 4.38^{* a, b}$ & $10.00 \pm 0.00^{* a, b}$ & $100.00 \pm 0.00^{*} \mathrm{a}, \mathrm{b}$ \\
\hline \multirow[t]{5}{*}{4} & S. gabonensis butanol fraction & 7.5 & 0.00 & 0.00 & 0.00 & 0.00 & 0.00 \\
\hline & & 15 & 0.00 & 0.00 & 0.00 & 0.00 & 0.00 \\
\hline & & 30 & 0.00 & 0.00 & 0.00 & 0.00 & $10.50 \pm 1.58^{* a, b}$ \\
\hline & & 60 & 0.00 & 0.00 & 0.00 & 0.00 & $9.50 \pm 1.58^{* a, b}$ \\
\hline & & 120 & 0.00 & 0.00 & 0.00 & 0.00 & $11.00 \pm 3.16^{* a, b}$ \\
\hline \multirow[t]{5}{*}{5} & S. gabonensis aqueous fraction & 7.5 & 0.00 & 0.00 & 0.00 & 0.00 & 0.00 \\
\hline & & 15 & 0.00 & 0.00 & 0.00 & 0.00 & 0.00 \\
\hline & & 30 & 0.00 & 0.00 & 0.00 & 0.00 & 0.00 \\
\hline & & 60 & 0.00 & 0.00 & 0.00 & 0.00 & 0.00 \\
\hline & & 120 & 0.00 & 0.00 & 0.00 & 0.00 & 0.00 \\
\hline \multirow[t]{2}{*}{6} & $\begin{array}{l}\text { Methyl 3,5-dihydroxy-4-meth- } \\
\text { oxybenzoate (Compound 1) }\end{array}$ & 7.5 & 0.00 & 0.00 & 0.00 & 0.00 & 0.00 \\
\hline & & 20 & 0.00 & 0.00 & 0.00 & 0.00 & 0.00 \\
\hline \multirow[t]{2}{*}{7} & Eriodictyol (Compound 2) & 7.5 & 0.00 & 0.00 & 0.00 & 0.00 & 0.00 \\
\hline & & 20 & 0.00 & 0.00 & 0.00 & 0.00 & 0.00 \\
\hline \multirow[t]{2}{*}{8} & Bergenin (Compound 3) & 7.5 & 0.00 & 0.00 & $60.50 \pm 3.69^{*} \mathrm{a}, \mathrm{b}$ & $61.00 \pm 3.94^{*} \mathrm{a}, \mathrm{b}$ & $61.00 \pm 3.94^{* a, b}$ \\
\hline & & 20 & 0.00 & 0.00 & $100.00 \pm 0.00$ & $100 \pm 0.00^{*} \mathrm{a}, \mathrm{b}$ & $100.00 \pm 0.00^{*} \mathrm{a}, \mathrm{b}$ \\
\hline \multirow[t]{2}{*}{9} & Normal saline & & & & & & \\
\hline & & & 0.00 & 0.00 & 0.00 & 0.00 & 0.00 . \\
\hline 10 & Cotton seed oil & 7.5 & $100.00 \pm 0.00$ & $100.00 \pm 0.00$ & $100.00 \pm 0.00$ & $100.00 \pm 0.00$ & $100.00 \pm 0.00$ \\
\hline
\end{tabular}

Data expressed as mean $\pm \operatorname{SEM}(n=10), * \mathrm{p}<0.05(* a$, when compared with normal saline and $* b$, when compared to cotton seed oil). 
From a previous report, it was found that activity was retained in the ethyl acetate fraction ${ }^{6}$ as was also confirmed in the in vitro spermicidal result. The isolated compound, bergenin showed 100\% spermicidal activity at $20 \mathrm{mg} / \mathrm{mL}$ concentration in 60 seconds while methyl 3,5-dihydroxy-4-methoxybenzoate and eriodictyol had no activity.

Compound 1 was isolated from Sacoglottis gabonensis as a yellow solid and identified as methyl 3,5-dihydroxy-4-methoxybenzoate (Fig. 1), with UV $\lambda_{\max } \mathrm{MeOH}_{260.5}$ and $298 \mathrm{~nm}$. The molecular formula was established as $\mathrm{C}_{9} \mathrm{H}_{10} \mathrm{O}_{5}$ based on spectral data obtained by ESI-MS. Positive and negative ESI-MS showed pseudo-molecular ion peaks at $\mathrm{m} / z$ (\% intensity) $199.0[\mathrm{M}+\mathrm{H}]^{+}(100)$ (base peak) and 197.1 $[\mathrm{M}-\mathrm{H}]^{-}(100)$ (base peak), respectively, indicating a molecular mass of $198 \mathrm{~g} / \mathrm{mol}$. This was further validated by the presence of the molecular ion peak at $m / z\left(\%\right.$ intensity) $394.8[2 \mathrm{M}+\mathrm{H}]^{-}(82)$ in the negative ESI-MS. The ${ }^{1} \mathrm{H}$ NMR $\left(\mathrm{CD}_{3} \mathrm{OD}\right): \delta(\mathrm{ppm})$ $7.02(2 \mathrm{H}, \mathrm{s}, \mathrm{H}-2, \mathrm{H}-6), 3.86\left(\mathrm{~s},-\mathrm{OCH}_{3}\right), 3.83$ (s, $-\mathrm{OCOCH}_{3}$ ). Comparison with literature data showed that it is methyl 3,5-dihydroxy-4-methoxybenzoate which was previously isolated from the flowers of Tamarix nilotica. ${ }^{9,10}$

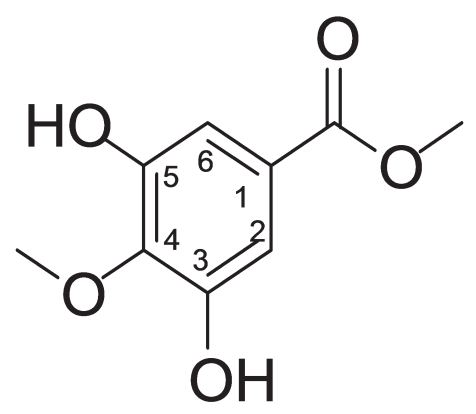

Figure 1. Methyl 3,5-dihydroxy-4-methoxybenzoate.

Compound 2 was isolated from Sacoglottis gabonensis as a light yellowish white solid and identified

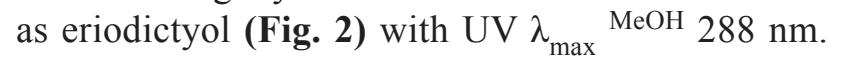
The molecular formula was established as $\mathrm{C}_{15} \mathrm{H}_{12} \mathrm{O}_{6}$ based on spectra data obtained by ESI-MS. Positive and negative ESI-MS showed pseudo-molecular ion peaks at $\mathrm{m} / z$ (\% intensity) $287.2[\mathrm{M}-\mathrm{H}]^{-}$(83) (base peak) and $289.2[\mathrm{M}+\mathrm{H}]^{+}(100)$ (base peak), respectively, indicating a molecular mass of 288 $\mathrm{g} / \mathrm{mol} .{ }^{1} \mathrm{H}$ NMR $\left(\mathrm{CD}_{3} \mathrm{OD}, 500 \mathrm{MHz}\right): \delta(\mathrm{ppm})$ 2.69 (H-3a, dd, J=3.0, $17.0 \mathrm{~Hz}), 3.06(\mathrm{H}-3 \mathrm{~b}, \mathrm{dd}$, $\mathrm{J}=12.8,17.0 \mathrm{~Hz}), 5.27(\mathrm{H}-2, \mathrm{~d}, \mathrm{~J}=12.8 \mathrm{~Hz}), 5.87$ (H-6, H-8, m), 6.79 (H-5, H-6', 2H, s) and 6.91 $\left(\mathrm{H}-2^{\prime}\right.$, s). SBE4D was identified as eriodictyol by comparison with literature data. ${ }^{11}$

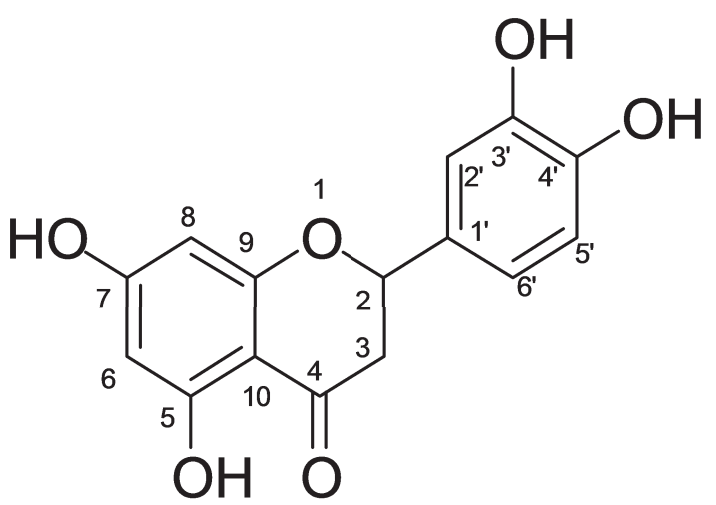

Figure 2. Eriodictyol.

Compound 3 was isolated from Sacoglottis gabonensis as a white crystalline solid and identified as Bergenin (Fig. 3), with UV $\lambda_{\max } \mathrm{MeOH}_{230}$ 288, and $328 \mathrm{~nm}$. The molecular formula was established as $\mathrm{C}_{14} \mathrm{H}_{16} \mathrm{O}_{9}$ based on spectral data obtained by ESI-MS. Positive and negative ESI-MS showed pseudo-molecular ion peaks at $\mathrm{m} / \mathrm{z}$ ( $\%$ intensity) $329.0[\mathrm{M}+\mathrm{H}]^{+}(100)$ (base peak) and $327.2[\mathrm{M}-$ $\mathrm{H}^{-}$(100) (base peak), respectively, indicating a molecular mass of $328 \mathrm{~g} / \mathrm{mol}$. This was further validated by the presence of the molecular ion peak at $\mathrm{m} / \mathrm{z}$ (\% intensity) $654.9[2 \mathrm{M}+\mathrm{H}]^{-}(20)$. The ${ }^{1} \mathrm{H}$ NMR $\left(\mathrm{CD}_{3} \mathrm{OD}, 500 \mathrm{MHz}\right): \delta(\mathrm{ppm}) 7.07$ $(\mathrm{H}-4, \mathrm{~s}), 4.95(\mathrm{H}-9, \mathrm{~d}, \mathrm{~J}=10.5 \mathrm{~Hz}), 3.68\left(2 \mathrm{H}, \mathrm{H}_{2}-\right.$ $16, \mathrm{~m}), 3.90\left(3 \mathrm{H}, \mathrm{s}, 6-\mathrm{OCH}_{3}\right), 3.44(\mathrm{H}-12, \mathrm{t}, \mathrm{J}=9.0$ $\mathrm{Hz}), 3.81(\mathrm{H}-13, \mathrm{t}, \mathrm{J}=9.0 \mathrm{~Hz}), 4.05(2 \mathrm{H}, \mathrm{m}, \mathrm{H}-11$, $\mathrm{H}-14)$. These values were consistent with those already reported for bergenin. ${ }^{12,13}$

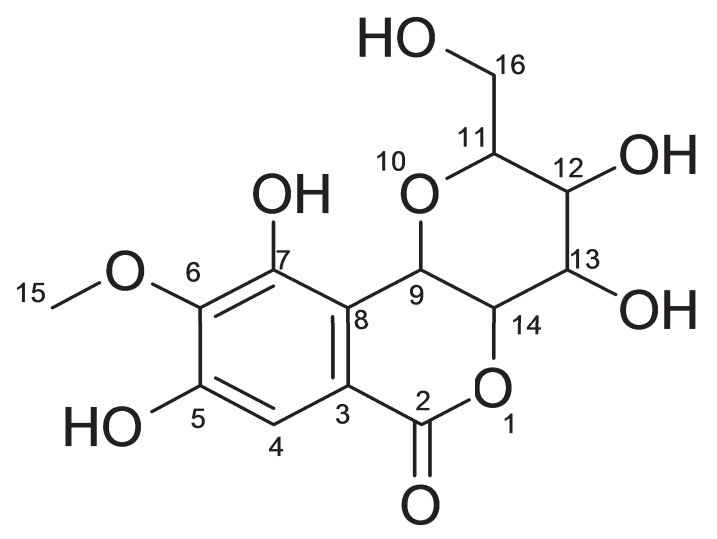

Figure 3. Bergenin.

\section{CONCLUSION}

The active spermicidal constituent in Sacoglottis gabonensis stem bark extract is bergenin. However, 
methyl 3,5-dihydroxy-4-methoxybenzoate and eriodictyol showed no activity. This plant is known for its aphrodisiac action; hence, caution may have to be exercised in its use because of its spermicidal effect.

\section{REFERENCES}

1. Gupta MD. Family planning program are effective. World Bank Research Observer 2014;29 (1):83-108.

2. Bell DL, Camacho EJ, Velasquez AB. Male access to emergency contraception in Pharmacies: a majority shopper survey. Contraception 2014;90(4):413-5.

3. Higgins JA, Smith NK, Sanders SA, et al. Dual method use at last sexual encounter: a nationally representative, episode-level analysis of US men and women. Contraception 2014;90 (4):399-406.

4. Roth MY, Shih G, Ilani N, et al. Acceptability of a transdermal gel-based male hormonal contraceptive in a randomized controlled trial. Contraception 2014;90(4):407-12.

5. Mali PC, Chaturvedi M, Dixit VP. Antispermatogenic activity of Solanum xanthocarpum Schrad \& Wandl. Root (50\% EtOH-extract) in rats. Journal of Phytol Research 1996;9(1):13-7.

6. Alade GO, Omobuwajo OR, Moody JO. Contraceptive effect of ethanolic extract of sacoglottis gabonensis (Bail.) Urb. Humiriaceae Stem Bark on male Wistar Rats. Inventi: Ethnopharmacology 2011;1:64-7.

7. Sander FV, Cramer SD. Practical method for testing the spermicidal action of chemical contraceptives. Hum Fertil 1941;6:134-7.

8. WHO (World Health Organization). Laboratory manual for the examination of human semen and spermcervical mucus interaction. 4th ed. Cambridge, New York: Cambridge University Press;1999. pp. 10-26.

9. Watanabe M, Fuda H, Jin S, et al. Isolation and characterization of a phenolic antioxidant from the Pacific oyster (Crassostrea gigas). J Agric Food Chem 2012;60(3):830-5.

10. Nawar MAM, Souleman AMA, Buddras J, et al. Phenolic constituents of the flowers of Tamarix nilotica: the structure of Nilocitin, a new Digalloylglucose. Tetrahedron Letters 1984;25(1):49-52.

11. Encarnacion DR, Nogueiras CL, Salinas VHA, et al. Isolation of Eriodictyol identical with Huazhongilexone from Solanum hindsianum. Acta Chemica Scandinavica 1999;53:375-7.

12. Nasser JA, Yaacob WA, Din LB, et al. Isolation of atranorin, bergenin and goniothalamin from Hopea sangal. ARPN Journal of Engineering and Applied Sciences 2009;4(1):92-5.

13. Nasir N, Koul S, Qurishi MA, et al. Evaluation of antioxidant and antimicrobial activities of Bergenin and its derivatives obtained by chemoenzymatic synthesis. Eur J Med Chem 2011;45:2415-20. 


\title{
Спермицидные ингредиенты экстракта этанола из коры стебля Sacoglottis gabonensis
}

\author{
Гидеон О. Аладе ${ }^{1,2}$, Джоунс О. Мууди르, Оланреваджу Р. Ауотона ${ }^{1}$, Сабури А. Адесаня ${ }^{3}$, \\ Даоуан Лаи 4 , Петер Прокш ${ }^{5}$ \\ ${ }^{1}$ Кафедра фармакогнозии и фитомедицины, Факультет фармации, Университет „Дельта Нигера“, Остров Уилберфорс, \\ Нигерия \\ 2 Кафедра фармакогнозии, Факультет фармации, Ибаданский университет, Ибадан, Нигерия \\ ${ }^{3}$ Кафедра фармакогнозии, Факультет фармации, Университет "Обафеми Аволово“, Иле-Ифе, Нигерия \\ ${ }^{4}$ Кафедра растительной патологии, Колледж защиты растений, Китайский аграрный университет, Пекин, НР Китай \\ ${ }^{5}$ Институт фармацевтической биологии и биотехнологий, Университет имени Генриха Гейне, Дюссельдорф, Германия
}

Адрес для корреспонденции: Гидеон О. Аладе, Кафедра фармакогнозии и фитомедицины, Факультет фармации, Университет „Дельта Нигера“, Амасома, Байелса Йенагоа, Байелса 560001, Нигерия

E-mail: aladegideon@yahoo.com Тел: +2348067368038

Дата получения: 05 июля 2016 Дата приемки: 22 марта 2017 Дата онлайн публикации: 09 мая 2017

Дата публикации: 22 декабря 2017

Ключевые слова: сперма, иммобилизация, ингредиент, Sacoglottis gabonensis

Образец цитирования: Alade GO, Moody JO, Awotona OR, Adesanya SA, Lai D, Proksch P. Spermicidal constituents of ethanolic extract of Sacoglottis gabonensis stem bark.

Folia Medica 2017;59(4):437-42. doi: 10.1515/folmed-2017-0047.
Цель: Целью настоящего исследования является изолирование спермицидных ингредиентов Sacoglottis gabonensis.

Материалы и методы: Экстракт этанола с измельчёнными частицами коры стебля Sacoglottis gabonensis был исследован на предмет установления иммобилизации спермы. Наиболее активная фракция этилацетата была дополнительно очищена с применением колоночной и полупрепаративной высокоэффективной жидкостной хроматографии, с целью изолирования ингредиентов, подлежащих характеристике спектроскопскими методами (UV, LC/MS, и NMR). Ингредиент был исследован на предмет установления активности иммобилизации спермы.

Результаты: Экстракт этанола проявил 100 \% активность иммобилизации спермы (р < 0.05) при концентрации 30 мг/мл. при 20с по сравнению как с положительными, так и с отрицательными контролями. Наиболее активной фракцией этилацетата является метил 3,5-дигидрокси-4-метоксибензоат, эриодиктиол и бергенин. Бергенин проявил 100 \% активность иммобилизации спермы при 20 мг/мл при 60с, что является значимым ( $<<0.05)$ также при сопоставлении с положительными и отрицательными контролями, в отличие от метил 3,5-дигидрокси-4-метоксибензоата и эриодиктиола, которые не показали активности.

Заключение: Активным спермицидным ингредиентом коры стебля Sacoglottis gabonensis является бергенин. Независимо от этого, метил 3,5-дигидрокси-4-метоксибензоат и изодиадиол не проявили активности. Данное растение известно как афродизиак и ввиду наличия спермицидных свойств надо проявлять осторожность при его применении. 